Pacific Journal of Mathematics

A NOTE ON A CONJECTURE OF HECK 


\title{
A NOTE ON A CONJECTURE OF HECKE
}

\author{
ARNOLd Pizer
}

\begin{abstract}
In 1940 Hecke made a conjecture concerning the representation of modular forms of weight 2 on $\Gamma_{0}(p), p$ a prime, by theta series. In this note we show that this conjecture is true if and only if $p<37$ or $p=41,47,59$, or 71 . We also propose a modified version of the conjecture which we have tested quite extensively.
\end{abstract}

1. Introduction. Hecke's conjecture (see [7], Satz 53 p. 884) states that a certain explicit set of theta series coming from quaternion algebras can be used to give a basis for the space of cusp forms of weight 2 on $\Gamma_{0}(p)$. Eichler knew in 1956 that Hecke's conjecture did not hold in general (see [3], p. 169). However, since there has been quite a bit of confusion on this point (see [5], p. 138 and [6], p. 148-9), we think it is worthwhile to reconsider the conjecture. More importantly, in Question 3.6 below, we propose a modified version of Hecke's conjecture which we have tested quite extensively on a computer.

Fix a prime $p$ and let $\mathfrak{A}$ denote the (unique definite) quaternion division algebra over $Q$, the field of rational numbers, ramified precisely at $p$ and $\infty$. Let $\mathcal{O}$ be a maximal order of $\mathfrak{A}$ and let $I$ be a left $\mathcal{O}$-ideal (see [5], Ch. 2 or [11]). We define the theta series $\theta_{I}(\tau)$, determined by $I$, by

$$
\Theta_{I}(\tau)=\sum_{z \in I} e^{2 \pi i \tau N(z))_{I}(I)}
$$

where $N(\quad)$ denotes the reduced norm of $\mathfrak{A}$ and $N(I)$ is the positive rational number that generates the fractional ideal of $Q$ generated by $\{N(a) \mid a \in I\}$. It is well known (see e.g., [5]) that $\Theta_{I}(\tau)$ is a modular form of weight 2 on $\Gamma_{0}(p)$. Let us denote by $S_{k}\left(\Gamma_{0}(p)\right)$ the space of cusp forms of weight $k$ on $\Gamma_{0}(p)$.

Let $\mathcal{O}$ be a maximal order of $\mathfrak{A}$ and let $I_{1}, \cdots, I_{H}$ be a complete set of representatives of the distinct left $\mathcal{O}$-ideal classes (we say two left $\mathcal{O}$-ideals $I$ and $J$ belong to the same class if and only if $I=J a$ for some $\left.a \in \mathfrak{Q}^{x}\right)$. It is well known that $H=H_{p}$, the class number, is finite and independent of the particular maximal order we choose (see [5] and [11]). Given two theta series $\Theta_{I}(\tau)$ and $\Theta_{J}(\tau), I$ and $J$ left $\mathcal{O}$-ideals, then $\Theta_{I}(\tau)-\Theta_{J}(\tau)$ is a cusp form (see Siegel [15], p. 376). Hecke's conjecture states that for any maximal order $\mathcal{O}$ and representatives $I_{1}, \cdots, I_{H}$ of the left $\mathcal{O}$-ideal classes, $\Theta_{I_{2}}(\tau)-\Theta_{I_{1}}(\tau), \cdots, \Theta_{I_{H}}(\tau)-\Theta_{I_{1}}(\tau)$ constitute a basis for $S_{2}\left(\Gamma_{0}(p)\right)$. One 
has formula's for $H_{p}$ (see Lemma 2.6 below) and for $\operatorname{dim} S_{2}\left(\Gamma_{0}(p)\right.$ ) (see [14]). The relation between them is $H_{p}=\operatorname{dim} S_{2}\left(\Gamma_{0}(p)\right)+1$. Hence Hecke's conjecture is equivalent to the $\Theta_{I_{1}}(\tau), \cdots, \Theta_{I_{H}}(\tau)$, $H=H_{p}$ being linearly independent and this is in fact almost the way Hecke stated the conjecture. His precise statement is as follows.

Let $\mathcal{O}$ be a maximal order in a quaternion algebra $\mathfrak{A} / Q$ ramified precisely at $p$ and $\infty$. Let $I_{1}, \cdots, I_{H}, H=H_{p}$ be a complete set of representatives of the distinct left $\mathcal{O}$-ideal classes. For all $j=1$, $\cdots, H, I_{j}^{-1} I_{j}=\mathscr{O}_{j}$ (say) is also a maximal order (see [5] or [11]) and

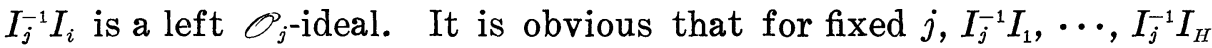
give a complete set of representatives of the distinct left $\mathscr{O}_{j}$-ideal classes. Let $\Theta_{i j}(\tau)=1 / e_{j} \Theta_{I_{j}^{-1} I_{i}}(\tau)$ where $e_{j}$ is the number of units of $O_{j}$. Consider the $H \times H$ matrix of theta series $\Theta=\left(\Theta_{i j}(\tau)\right)$. Hecke's precise conjecture (see [7], p. 884) is that

(2) the theta series in any one column of the matrix $\Theta$ are linearly independent

and hence they generate the space of cusp forms of weight 2 on $\Gamma_{0}(p)$ plus a 1 dimensional space of noncusp forms. Note that we can write $\Theta=\left(\Theta_{i j}(\tau)\right)=\sum_{n=0}^{\infty} B(n) e^{2 \pi i n \tau}$ with $H \times H$ rational (in fact integral if $n>0$ ) matrices $B(n)$.

The $B(n)$ are the Brandt Matrices and they give the action of the Hecke operators $T(n),(n, p)=1$ on the $\Theta_{i j}(\tau)$ (see [5], p. 138). As any maximal order is isomorphic (i.e., conjugate) to some $\mathscr{O}_{i}$ (see Lemma 2.1 below) and as the theta series corresponding to a maximal order depend only on the isomorphism class of that order (see Lemma 2.7 below), we see that all our versions of Hecke's conjecture are equivalent.

In 1956 Eichler [3] proved that if one diagonalizes the matrix $\Theta$ (i.e., if one simultaneously diagonalizes the $B(n)$ ), then one of the resulting diagonal entries of the diagonalized matrix will be the zeta function of the algrebra $\mathfrak{A}$ (and hence not a cusp form) and the remaining $H-1$ diagonal entries will be cusp forms (in fact eigen forms for all $T(n),(n, p)=1$ ) and give a basis for $S_{2}\left(\Gamma_{0}(p)\right.$ ). Hence at least in spirit, if not in fact, Hecke's conjecture is true for $\Gamma_{0}(p)$. Eichler's results have now been generalized to the case of cusp forms of weight $k \geqq 2$ on $\Gamma_{0}(M), M$ not a perfect square by Eichler [5], Hijikata-Saito [8], and Pizer [10]. In essence these generalizations show that all new forms of weight $k \geqq 2$ on $\Gamma_{0}(M), M$ not a square, can be obtained from (generalized if $k>2$ ) theta series attached to orders of "level" $M$ in a rational quaternion algebra.

However, Hecke's original conjecture (2) is still of some interest. In fact there seems to have been some confusion as to whether 
Eichler's results established the original conjecture (see [5], p. 138 and [6], p. 148-9 and also [13], p. 139) but as Eichler knew (see [3], p. 169) they do not. We give below two easy proofs that the conjecture can not hold in general and then ask if a modified version of Hecke's conjecture might be true.

2. Arithmetic of quaternion algebras. We state in a series of lemmas those results from the arithmetic of quaternion algebras that we require. Most are well known and easy to prove. We refer to references for most of the proofs. The reference Eichler [2] or [5] will usually provide a proof in the case at hand and the reference Pizer [11] will usually give the generalization of the result needed to consider the case $\Gamma_{0}(M), M$ not a perfect square. In this section we fix a prime $p$ and a quaternion algebra $\mathfrak{A} / Q$ ramified precisely at $p$ and $\infty$.

Lemma 2.1. Let $\bigcirc$ be a maximal order $\mathfrak{A}$ and let $I_{1}, \cdots, I_{H}$ be a complete set of representatives of the distinct left $\mathcal{O}_{\text {-ideal classes. }}$ Then the right orders $\mathcal{O}_{i}=\left\{a \in \mathfrak{A} \mid I_{i} a \subset I_{i}\right\}=I_{i}^{-1} I_{i}$ of the $I_{i}$ are maximal and represent (with possible duplication) all the types (i.e., isomorphism classes) of maximal orders of $\mathfrak{A}$.

Proof. See [2] p. 132 and p. 136 or [11], Prop. 2.15.

Definition 2.2. A left $\mathcal{O}$-ideal $I$ is said to be a two-sided $\mathcal{O}$ ideal if its right order is also $O$.

It is clear that if we fix $\mathscr{O}$ the set of two-sided $\mathscr{O}$-ideals forms a group. If $I$ and $J$ are two sided $\mathcal{O}$-ideals and $I=J a, a \in \mathfrak{V}^{x}$, then $\mathscr{O}=a^{-1} \mathscr{O} a$ as $I$ and $J a$ have the same right order and thus $\mathscr{O} a$ is also a two-sided $O$-ideal. Hence we can consider the ideal class group of two-sided $\mathcal{O}$-ideals, i.e., the group of all two-sided $\mathcal{O}$-ideals module principal two-sided $\mathcal{O}^{-i d e a l s .}$

Lemma 2.3. Let $\mathcal{O}$ be a maximal order of $\mathfrak{A}$. Then the ideal class group of two-sided O-ideals has order 1 or 2.

Proof. See [5] p. 92 or [11], Cor. 2.21.

DeFinition 2.4. The Type number $T=T_{p}$ of the quaternion algebra $\mathfrak{A}$ (ramified at $p$ and $\infty$ ) is the number of isomorphism classes of maximal orders of $\mathfrak{A}$.

Lemma 2.5. Fix a maximal order 0 and let $I_{1}, \cdots, I_{T}, T=T_{p}$

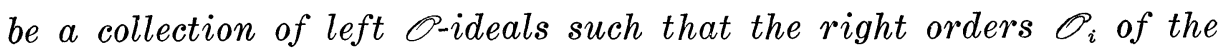


$I_{i}$ give a complete set of distinct representatives of the types of maximal orders (see Lemma 2.1). Let $h_{i}$ denote the ideal class number of two-sided $\mathcal{O}_{i}$-ideals. Assume the $I_{i}$ have been so arranged that $h_{i}=2$ for $i=1, \cdots, r$ and $h_{i}=1$ for $i=r+1, \cdots, T$. If $h_{i}=2$, let $J_{i}$ be a nonprincipal two-sided $\mathcal{O}_{i}$-ideal. Then $I_{1}, I_{1} J_{1}, I_{2}$, $I_{2} J_{2}, \cdots, I_{r}, I_{r} J_{r}, I_{r+1}, \cdots, I_{T}$ give a complete set of representatives of the distinct $O_{\text {-ideal classes. }}$

Proof. See [2] p. 136 or [11], Prop. 2.18.

LEMMA 2.6. Let the notation be the same as in Lemma 2.5. Then we have

(a) $H=H_{p}=1 / 12(p-1)+1 / 4(1-(-4 / p))+1 / 3(1-(-3 / p))$

(b) $T_{2}=T_{3}=1$

$$
\begin{aligned}
& T=T_{p}=1 / 2 H p+2^{-\tau(p)-1} h(\sqrt{-p)} \text { for } p>3 \\
& \text { where } \tau(p)=\left\{\begin{array}{rll}
1 & \text { if } p \equiv 1(4) \\
-1 & \text { if } & p \equiv 3(8) \\
0 & \text { if } & p \equiv 7(8)
\end{array}\right.
\end{aligned}
$$

and where $h(\sqrt{-p)}$ is the class number of $Q(\sqrt{-p)}$

(c) $H=\sum_{i=1}^{T} h_{i}$.

(d) $1 / 2 H_{p}<T_{p} \leqq H_{p}$ for all $p$.

(e) $T_{p}=H_{p}$ if and only if $p<37$ or $p=41,47,59$, or 71 .

(f) not all $h_{i}=2$ for any $p$.

(g) all $h_{i}=1$ if and only if $T_{p}=H_{p}$.

Proof.

(a) See [5] p. 113.

(b) See [1] p. 29 or [9] p. 93-94.

(c) Obvious from Lemma 2.5.

(d) Obvious from (b) and (c).

(e) It is clear from (b) that equality can hold for only small $p$ as $\left(h \sqrt{-p)} \sim\left(\operatorname{disc}(Q(\sqrt{-p)}))^{1 / 2} \log (p)\right.\right.$ and for these we verify (e) directly using (a) and (b).

(f) Obvious from (c) and (d).

(g) Clear from (c).

Lemma 2.7. Let $I$ be a left $\mathcal{O}^{-i d e a l}$ and let $a, b \in \mathfrak{Q}^{x}$. Then $\Theta_{a I b}(\tau)=\Theta_{I}(\tau)$. 


$$
\begin{aligned}
\Theta_{a I b}(\tau) & =\sum_{z \in a I b} e^{2 \pi i \tau N(z) / N(a I b)} \\
& =\sum_{z \in I} e^{2 \pi i \tau N(a z b) / N(a I b)} \\
& =\sum_{z \in I} e^{2 \pi i N(z) / N(I)} \\
& =\Theta_{I}(\tau)
\end{aligned}
$$

3. Hecke's conjecture. Fix a prime $p$ and a definite quaternion division algebra ramified precisely at $p$ and $\infty$.

Proposition 3.1. Let $\mathcal{O}$ be a maximal order of $\mathfrak{A}$ with no nonprincipal two-sided ideals. Let $I_{1}, \cdots, I_{H}, H=H_{p}$ be a complete set of representatives of the distinct left O-ideal classes. Then at most $T=T_{p}$ of the theta series $\Theta_{I_{1}}(\tau), \cdots, \Theta_{I_{H}}(\tau)$ are distinct.

Proof. By Lemma 2.7, the set of theta series $\Theta_{I_{1}}(\tau), \cdots, \Theta_{I_{H}}(\tau)$ is independent of the particular set of ideal class representatives we choose. Thus let $I_{1}, I_{1} J_{1}, \cdots, I_{r}, I_{r} J_{r}, I_{r+1}, \cdots, I_{T}$ be a set of ideal class representatatives as in Lemma 2.5. Here $J_{i}$ is a nonprincipal twosided $\mathscr{O}_{i}=I_{i}^{-1} I_{i}$ ideal. Let $J_{j}^{\prime}=I_{i} J_{i} I_{i}^{-1}$. Then $J_{i}^{\prime} I_{i}=I_{i} J_{i}$ and $J_{i}^{\prime}$ is a two-sided $\mathcal{O}$-ideal, hence principal, i.e., $J_{i}^{\prime}=a \mathscr{O}$ for some $a \in \mathfrak{A}^{x}$. Thus $\Theta_{I_{i} J_{i}}(\tau)=\Theta_{J_{i}^{\prime} I_{i}}(\tau)=\Theta_{a I_{i}}(\tau)=\Theta_{I_{i}}(\tau)$ by Lemma 2.7 and hence at most $T$ of the theta series can be distinct.

THEOREM 3.2. The following are equivalent

(i) Hecke's conjecture (2) is valid for $\Gamma_{0}(p)$.

(ii) $T_{p}=H_{p}$.

(iii) $p<37$ or $p=41,47,59$, or 71 .

Proof. (i) $\Rightarrow$ (ii): immediate from Lemma 3.6, part $f$ and Proposition 3.1.

(ii) $\Rightarrow$ (iii): this is $e$ of Lemma 2.6 .

(iii) $\Rightarrow$ (i ): we have developed a computer algorithm for generating the Brandt Matrices (see [12]). Direct verification shows that Hecke's conjecture holds for $p<37$ and $p=41,47,57$, and 71 . Note that Hecke himself checked this for $p<37$ (and also supposedly for $p=37$ ), see [7], p. 884 .

We give another proof that Hecke's conjecture can not hold in general.

Proposition 3.3. Let $\mathcal{O}$ be a maximal order of $\mathfrak{A}$ with no nonprincipal two-sided ideals. Let $I_{1}, \cdots, I_{H}, H=H_{p}$ be a complete set of representative of the left $\mathcal{O}^{-i d e a l}$ classes. Then $\Theta_{I_{1}}(\tau), \cdots$, $\Theta_{I_{H}}(\tau)$ can be linearly independent only for finitely many primes 
p. Here, of course, $\mathfrak{A}$ is the quaternion algebra ramified precisely at $p$ and $\infty$.

Proof. Let $E=\left(\begin{array}{rr}0 & -1 \\ p & 0\end{array}\right)$ denote the canonical involution on modular forms of weight 2 on $\Gamma_{0}(p)$. Then by Theorem 3.2 of [11], $\left.\Theta_{I_{i}}\right|_{E}(\tau)=$ $-\Theta_{\widetilde{M}_{I_{i}}}(\tau)$ where $\tilde{M}$ is a canonical two-sided $\mathcal{O}$-ideal. But $\tilde{M}$ must be principal, say $\tilde{M}=a \bigcirc$ for some $a \in \mathfrak{A}^{x}$. Thus $\left.\Theta_{I_{i}}\right|_{E}(\tau)=-\Theta_{a I_{i}}(\tau)=$ $-\Theta_{I_{i}}(\tau)$. If the $\Theta_{I_{i}}(\tau)$ are linearly independent, they span the space of cusp forms and hence the canonical involution $E$ is multiplication by -1 on $S_{2}\left(\Gamma_{0}(p)\right)$. But the fact that this is not true in general follows from the existence of formulas which give the dimension of the -1 and +1 eigen spaces of $E$ on $S_{2}\left(\Gamma_{0}(p)\right.$ ) (see [1]).

REMARK 3.4. As mentioned above we have developed an algorithm for generating the Brandt Matrices $B(n)$ in the case $S_{2}\left(\Gamma_{0}(M)\right), M$ not a perfect square. Looking at some computations gave many counter examples to Hecke's original conjecture and led very quickly to our results and also to the modified Hecke conjecture given below. We will publish an outline of the algorithm in the future (see [12]).

REMARK 3.5. In all our computations the following has been observed. If the maximal order $\mathcal{O}$ has a nonprincipal two-sided ideal (hence Proposition 3.1 does not apply), the $H$ theta series $\Theta_{I_{1}}(\tau)$, $\cdots, \Theta_{I_{H}}(\tau)$ have always been linearly independent. Here $I_{1}, \cdots, I_{H}$ are representatives of the left $\mathcal{O}$-ideal classes. If all the two-sided $\mathcal{O}$-ideals are principal (hence Proposition 3.1 does apply), the number of linearly independent theta series among the $\Theta_{I_{i}}(\tau), \cdots, \Theta_{I_{H}}(\tau)$ has always been $T$. That is the failure of Hecke's conjecture in all our examples has been completely explained by Proposition 3.1. This raises the obvious.

Question 3.6. Is the following modified version of Hecke's conjecture valid? Let $\mathcal{O}$ be a maximal order in a quaternion algebra $\mathfrak{A}$ over $Q$ ramified precisely at $p$ and $\infty$. Let $I_{1}, \cdots I_{H}$ be a complete set of representatives for the distinct left $\mathcal{O}$-ideal classes and let $\Theta_{I_{1}}(\tau), \cdots, \Theta_{I_{H}}(\tau)$ be the corresponding theta series. If $\odot$ has nonprincipal two-sided ideals (i.e., if the "prime ideal" of $O$ lying above $p$ is nonprincipal), are the $\Theta_{I_{1}}(\tau), \cdots, \Theta_{I_{H}}(\tau)$ always linearly independent? Further, if all two-sided $\mathcal{O}$-ideals are principal, are $T$ (the type number) of the $\Theta_{I_{1}}(\tau), \cdots, \Theta_{I_{H}}(\tau)$ always linearly independent?

REMARK 3.7. All our results have immediate analogues in the case $\Gamma_{0}(M), M$ not a perfect square. The arithmetic of quaternion 
algebras necessary to carry over these analogues is given in [11]. It should be noted that in general for $\Gamma_{0}(M)$ it may happen that for all orders $\mathcal{O}$ of "level $M$ " in a fixed quaternion algebra $\mathfrak{A}$ the theta series $\Theta_{I_{1}}(\tau), \cdots, \Theta_{I_{H}}(\tau)$ are linearly dependent. Here of course $I_{1}, \cdots, I_{H}$ is a complete set of representatives of the left $\mathcal{O}$-ideal classes. In fact $\Gamma_{0}(30)\left(M=30, q_{1}=3, q_{2}=10\right)$ provides an example where this happens.

REMARK 3.8. It might be interesting to note that in [1] p. 30-31 Deuring shows how the canonical involution acts on theta series. Then by assuming Hecke's conjecture he derives the formulas for the dimension of the eigen spaces of $E$ which by our Proposition 3.3 show that Hecke's conjecture can not hold, at least not in its full generality. The fact that Deuring obtains the correct formulas, however, may be taken as evidence that for some maximal order having nonprincipal two-sided ideals, the corresponding theta series are linearly independent.

\section{REFERENCES}

1. M. Deuring, Die Anzahl der Typen von Maximalordnungen einer definiten Quaternionenalgebra mit primer Grundzahl, Jber. Deutsch, Math. Verein., 54 (1950), 24-41. 2. M. Eichler, Zur Zahlentheorie der Quaternionen-Algebren, J. reine angew. Math., 195 (1955), 127-151.

3. - Uber die Darstellbarkeit von Modulformen durch Thetareichen, J. reine und angew. Math., 195 (1955), 156-171.

4. - Quadratische Formen und Modulfunktionen, Acta Arith., 4 (1958), 217239 .

5. - The basis problem for modular forms and the traces of the Hecke operators, Lecture Notes on Math., \#320, Springer-Verlag, 75-151.

6. - Corrigenda, Lecture Notes in Math., \#476, Springer-Verlag, 145-147.

7. E. Hecke, Analytische Arithmetik der positiven quadratischen Formen, Math. Werke, 789-918.

8. H. Hijikata and H. Saito, On the representability of modular forms by theta series, Number Theory, Algebraic Geometry and Commutative Algebra in honor of Y. Akizuki, Kinokuniya, Tokyo, (1973), 13-21.

9. A. Pizer, Type numbers of Eichler orders, J. reine angew. math., 264 (1973), 76-102.

10. - The representability of modular forms by theta series, J. Math. Soc. of Japan, 28 (1976), 689-698.

11. The action of the canonical involution on modular forms of weight 2 on $\Gamma_{0}(M)$, Math. Ann., 226 (1977), 99-116.

12. - An algorithm for computing modular forms on $\Gamma_{0}(N)$, to appear.

13. C. Siegel, Ober die analytische Theory der quadratic Formen, Gesammelte Abhandlunge, Band I, Springer-Verlag, (1966).

14. P. Ponomarev, A correspondence between quaternary quadratic forms, Nagoya Math. J., 62 (1976), 125-140.

15. G. Shimura, Introduction to the Arithmetic Theory of Automorphic Functions, Princeton Univ. Press, 1971. 
Received February 25, 1977. Research partially supported by NSF Grant MCS 7408108-A02.

UNIVERSITY OF ROCHESTER

ROCHESTER, NY 14627 


\section{PACIFIC JOURNAL OF MATHEMATICS}

\section{EDITORS}

RichaRd ARENS (Managing Editor)

University of California

Los Angeles, CA 90024

Charles W. Curtis

University of Oregon

Eugene, OR 97403

C. C. Moore

University of California

Berkeley, CA 94720

\section{J. DUGUNDJI}

Department of Mathematics

University of Southern California

Los Angeles, CA 90007

R. FinN and J. Milgram

Stanford University

Stanford, CA 94305

\section{E. F. BECKENBACH \\ B. H. NeumanN

\author{
UNIVERSITY OF BRITISH COLUMBIA \\ CALIFORNIA INSTITUTE OF TECHNOLOGY \\ UNIVERSITY OF CALIFORNIA \\ MONTANA STATE UNIVERSITY \\ UNIVERSITY OF NEVADA, RENO \\ NEW MEXICO STATE UNIVERSITY \\ OREGON STATE UNIVERSITY \\ UNIVERSITY OF OREGON
}

F. WOLF

K. Yoshida

\section{SUPPORTING INSTITUTIONS}

\author{
UNIVERSITY OF SOUTHERN CALIFORNIA \\ STANFORD UNIVERSITY \\ UNIVERSITY OF HAWAII \\ UNIVERSITY OF TOKYO \\ UNIVERSITY OF UTAH \\ WASHINGTON STATE UNIVERSITY \\ UNIVERSITY OF WASHINGTON
}

The Supporting Institutions listed above contribute to the cost of publication of this Journal, but they are not owners or publishers and have no responsibility for its content or policies.

Mathematical papers intended for publication in the Pacific Journal of Mathematics should be in typed form or offset-reproduced, (not dittoed), double spaced with large margins. Please do not use built up fractions in the text of the manuscript. However, you may use them in the displayed equations. Underline Greek letters in red, German in green, and script in blue. The first paragraph or two must be capable of being used separately as a synopsis of the entire paper. Items of the bibliography should not be cited there unless absolutely necessary, in which case they must be identified by author and journal, rather than by item number. Manuscripts, in triplicate, may be sent to any one of the editors. Please classify according to the scheme of Math. Reviews, Index to Vol. 39. All other communications should be addressed to the managing editor, or Elaine Barth, University of California, Los Angeles, California, 90024.

50 reprints to each author are provided free for each article, only if page charges have been substantially paid. Additional copies may be obtained at cost in multiples of 50 .

The Pacific Journal of Mathematics is issued monthly as of January 1966. Regular subscription rate: $\$ 72.00$ a year (6 Vols., 12 issues). Special rate: $\$ 36.00$ a year to individual members of supporting institutions.

Subscriptions, orders for numbers issued in the last three calendar years, and changes of address should be sent to Pacific Journal of Mathematics, P.O. Box 969, Carmel Valley, CA 93924, U.S.A. Older back numbers obtainable from Kraus Periodicals Co., Route 100, Millwood, NY 10546.

PUBLISHED BY PACIFIC JOURNAL OF MATHEMATICS, A NON-PROFIT CORPORATION

Printed at Kokusai Bunken Insatsusha (International Academic Printing Co., Ltd.). 8-8, 3-chome, Takadanobaba, Shinjuku-ku, Tokyo 160, Japan. 


\section{Pacific Journal of Mathematics \\ Vol. 79, No. $2 \quad$ June, 1978}

David R. Adams, Quasi-additivity and sets of finite $L^{p}$-capacity ........ 283

George M. Bergman and Warren Dicks, Universal derivations and universal

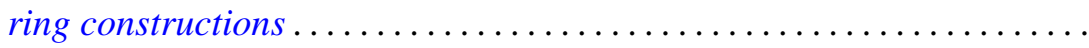

Robert F. Brown, Addendum to: "Fixed points of automorphisms of compact Lie groups".........................................

Eugene Frank Cornelius, Jr., Characterization of a class of torsion free

groups in terms of endomorphisms .......................

Andres del Junco, A simple measure-preserving transformation with trivial centralizer..................................... 357

Allan Lee Edmonds, Extending a branched covering over a handle ...... 363

Sjur Flam, A characterizaton of $\mathbf{R}^{2}$ by the concept of mild convexity .......

Claus Gerhardt, $L^{p}$-estimates for solutions to the instationary Navier-Stokes

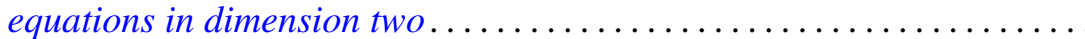

Kensaku Gomi, Finite groups with a standard subgroup isomorphic to

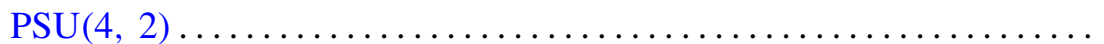

E. E. Guerin, A convolution related to Golomb's root function ........... 463

$\mathrm{H}$. B. Hamilton, Modularity of the congruence lattice of a commutative cancellative semigroup .................................

Stephen J. Haris, Complete reducibility of admissible representations over function fields.

Shigeru Itoh and Wataru Takahashi, The common fixed point theory of singlevalued mappings and multivalued mappings... ...

James E. Joseph, Multifunctions and graphs .............

Bruce Magurn, Images of $S K_{1} Z G$. .

Arnold Koster Pizer, A note on a conjecture of Hecke... .

Marlon C. Rayburn, Maps and h-normal spaces ...........

Barada K. Ray and Billy E. Rhoades, Corrections to: "Fixed-point theorems for mappings with a contractive iterate" ..............

Charles Irvin Vinsonhaler, Corrections to: "Torsion free abelian groups quasiprojective over their endomorphism rings. II". . . 